\title{
Efficient High-speed Current-mode Links for Network-on-Chip Performance Optimization
}

\author{
Hamed S. Kia and Cristinel Ababei \\ Department of Electrical and Computer Engineering \\ North Dakota State University, Fargo ND, USA \\ Email: \{hamed.sajjadikia, cristinel.ababei\}@ndsu.edu
}

\begin{abstract}
We propose a novel cost-effective long-range NoC interconnect design based on current-mode signaling. The proposed CMOS based long-range link reduces the communication delay of long wires significantly without using traditional pipelined repeaters, making it a cost efficient alternative to wireless and optical interconnects. Spice simulations are performed to verify the performance of the proposed current-mode transceiver. The performance of the proposed interconnect is analyzed and compared with wireless, optical and existing CMOS based interconnects. Also a modified NoC simulator is utilized to demonstrate the benefit of current-mode long-range links.
\end{abstract}

\section{INTRODUCTION}

Continuous technology downscaling enabled increased device densities and improved device performance. However, downscaling also lead to an increase of the resistance and capacitance per unit length for interconnects, which in turn resulted into a shift of the circuit delay bottleneck from logic gates to wires. This shift is further accelerated by the trend of global interconnects to become longer due to the increase in maximum die size. Early solutions to this global delay problem included pipelining of the interconnects via repeater insertion. However, repeater insertion is not a scalable or sustainable solution anymore. One of the most promising recent solutions to address the global delay problem and the need for higher communication concurrency in today's complex systems-onchip (SoCs) including multiprocessor systems-on-chip (MPSoCs) is the network-on-chip ( $\mathrm{NoC})$ [1], [2]. NoCs replace the traditional global on-chip buses and provide a generic onchip interconnection network realized by specialized routers or switches connected via short-range links. While the delay of these short-range links continue to represent a design challenge, it is the latency between remote network routers that is of even higher concern. This problem can be addressed via insertion of long-range links that can be implemented via traditional interconnects with repeaters. Since the idea of longrange link insertion has been introduced in the context of NoCs [3], several different types of implementations have been proposed. These implementations can be basically classified into three main classes: wireless, optical, and CMOS wires. Optical interconnects require converting signals between optical and electrical domains, an increased area, and have to deal with fabrication technology incompatibility. Wireless interconnects have issues related to extra area and power consumption of the transceivers. Yet another recent alternative solution is currentmode signaling. Current-mode links are fully CMOS and can achieve nearly speed-of-light speed. Their main drawback is the area penalty needed to implement transceivers that implement voltage-to-current and current-to-voltage converters.

As mentioned above, one of the alternatives to the traditional metal interconnects is wireless interconnects. The network architecture is augmented with wireless nodes spread across the chip. These nodes form a wireless network superimposed with the regular mesh network, which can be employed to transmit data via wireless paths with smaller number of hops compared to the regular mesh network. For example, RF interconnect shortcuts have been used to design novel NoC reconfigurable architectures in [4]. A simultaneous tri-band on chip RF interconnect for NoCs was reported in [5], where two RF bands in mm-wave frequencies were modulated using amplitude-shift keying.

Optical or photonic interconnects integrate one or several high bandwidth optical links that span the whole NoC area to provide high speed links between remote network routers. Because of the potentially significant performance benefits that optical communications can offer a lot of research concentrated on this area. For example, an optical $\mathrm{NoC}(\mathrm{ONoC})$ was designed and investigated in [9]. A hybrid micro-architecture for NoCs that combines a broadband photonic circuit-switched network with an electronic packet-switched control network is described in [10]. [11] combines photonic rings on multiple photonic layers within 3D mesh NoC architectures. Other recent studies on optical interconnects and their use within NoC based systems as well as network simulators include [12]-[21].

Most of the traditional CMOS interconnects work in voltage-mode. While they are cheap to fabricate, they suffer from the rapid increase of their delay with length. This problem is typically addressed via interconnect pipelining with repeaters or buffers, which translate into area penalty. An alternative solution is offered by several earlier proposals for interconnects whose operation is in current-mode. Currentmode links operate with currents as the main information carriers while voltage variation is limited to narrow ranges. As a result, current-mode links can achieve nearly speedof-light latencies. In addition, current-mode transceivers are entirely CMOS thereby cost-effective and easy to fabricate. Their main disadvantage compared to voltage-mode links is that they require transceivers that may result into larger area penalty compared to that of repeaters of the voltage-mode 
links. One of the earlier current-mode link proposals for onchip interconnects is [22]. A new transceiver design based on differential current-mode sensing to reduce both delay and energy dissipation compared to the standard inverter repeater approach is proposed in [23]. Authors in [24] use a sharp current-pulse data transmission to modulate transmitter energy to higher frequencies, where the effect of wire inductance is maximized, allowing the on-chip wires to function as transmission lines with considerably reduced dispersion. [25] reports an asynchronous serial link for on-chip communication as an alternative to standard bit-parallel links. The serial interconnect uses a differential level encoded dual-rail twophase asynchronous protocol, and avoids per-bit handshake and eliminating per-bit synchronization. A high-performance long-range NoC link design based on multilevel current-mode signaling and delay insensitive two-phase 1-of-4 encoding is studied in [26]. This design achieves higher throughput and lower latency compared to voltage-mode interconnects.

In this paper, we focus on CMOS based current-mode links that represent a compromise between the high performance of the wireless and optical interconnects and the reduced area and cost of the voltage-mode interconnects. We propose a new current-mode link with the main objective of minimizing the area of the transceiver without sacrificing performance.

The remainder of this paper is organized as follows. In the next section, we introduce the proposed current-mode link design. Section III reports simulation results. In Section IV, we study the use of the proposed current-mode link as long-range links in NoCs. We conclude in Section V by summarizing our main contribution.

\section{Proposed Current-Mode Link}

In this section, we introduce the current-mode link design that represents the main contribution of this paper. Because the main application of the proposed current-mode link is the implementation of long-range links in NoCs, our primary goal is to develop a cost effective solution without compromising the link performance. Cost effective current-mode links are desirable to minimize the area increase of network routers compared to the traditional router architectures. Earlier efforts on current-mode links used differential solutions [24] that were motivated by noise or architectural constraints. The overall current-mode link architecture is shown in Fig.1.

The transmitter is implemented using a current-mode differential driver [27] formed basically by the two PMOS transistors $M 1$ and $M 2$, which are controlled by the outputs $Q$ and $\bar{Q}$ of the flip-flop that in turn is driven by the pulses of the input voltage $V_{i n}$. During normal operation, when $V_{i n}$ is logic 1, the transistor $M 1$ turns off while transistor $M 2$ turns on to direct the current $I_{0}$ to resistor $R 2$ and the actual link. On the other hand, when $V_{i n}$ is logic 0, M2 turns off while $M 1$ turns on to drive current $I_{0}$ to resistor $R 1$ and away from the link.

The receiver is formed by four transistors $M 3, M 4, M 5$ and $M 6$, a Schmitt trigger, and two inverters as shown in Fig.1. The key point of the receiver architecture is that the voltage swing of $V_{s}$ is limited to a small range through the action of the Schmitt trigger and the feedback created via transistors $M 5$ and $M 6$. The two inverters regenerate the output from the Schmitt trigger to provide the output voltage $V_{\text {out }}$ and to drive the inverter formed by $M 5$ and $M 6$. The small voltage swing of $V_{s}$ is essential to achieving a fast generation of the output voltage $V_{\text {out }}$. The range for the variation of $V_{s}$ is centered at a reference voltage $V_{\text {ref }}$ determined by $M 3$ and $M 4 . V_{\text {ref }}$ is selected such that it is in the middle of the voltage range given by the low and high threshold voltages of the Schmitt trigger, $V_{T L}$ and $V_{T H}$ (see also Fig.2).

In the following subsections, we describe the operation of the proposed current-mode link when either a logic 1 or a logic 0 is transmitted.

\section{A. Operation when logic 1 is transmitted}

When the input voltage $V_{i n}$ is logic 1, the differential pair $M 1$ and $M 2$ directs all current $I_{0}$ to resistor $R 2$ and the actual link. $R_{2}$ is selected such that most of the current flows into the link. In our analysis, we model the physical link as a distributed RC transmission line whose technology parameters can be estimated via technology prediction models.

Once the current $I_{0}$ is directed to the link, the equivalent capacitance of the link and its load starts charging. In other words, the net equivalent capacitance of the node $V_{s}$ is getting charged and $V_{s}$ starts to increase until it reaches the upper threshold voltage $V_{T H}$ of the Schmitt trigger. At this moment, the output of the Schmitt trigger goes high, which in turn results into the output voltage $V_{\text {out }}$ to become logic 1. Once $V_{\text {out }}$ switches to logic 1, the feedback formed by the transistors $M 5$ and $M 6$ helps to stop the increase of $V_{s}$ by pulling (via M5) some of the current that charges the node $V_{s}$. This operation is illustrated for example in the fifth clock period or cycle shown in Fig.2.

\section{B. Operation when logic 0 is transmitted}

On the other hand, when the input voltage $V_{i n}$ is logic 0 , the differential pair $M 1$ and $M 2$ directs all current $I_{0}$ to resistor $R 1$. In this case, no current drives the physical link. As a result, the voltage $V_{s}$ starts to decrease until it reaches the lower threshold voltage $V_{T L}$ of the Schmitt trigger, which triggers and changes its output to low. This in turn results into the output voltage $V_{\text {out }}$ to become logic 0 . Once this happens, the feedback formed by the transistors $M 5$ and $M 6$ helps to stop the decrease of $V_{s}$ by pushing (via $M 6$ ) current to sustain the node $V_{s}$. This operation is illustrated for example in the seventh clock period shown in Fig.2.

\section{EXPERIMENTAL Results AND Performance ANALYSIS}

To test the performance of the proposed current-mode link we implemented the transceiver shown in Fig.1 using $0.18 \mu m$ CMOS technology parameters. The $0.18 \mu m$ technology was chosen intentionally because we wanted to compare our proposed interconnect with previous work. The actual physical link is modeled as a distributed RC transmission line 


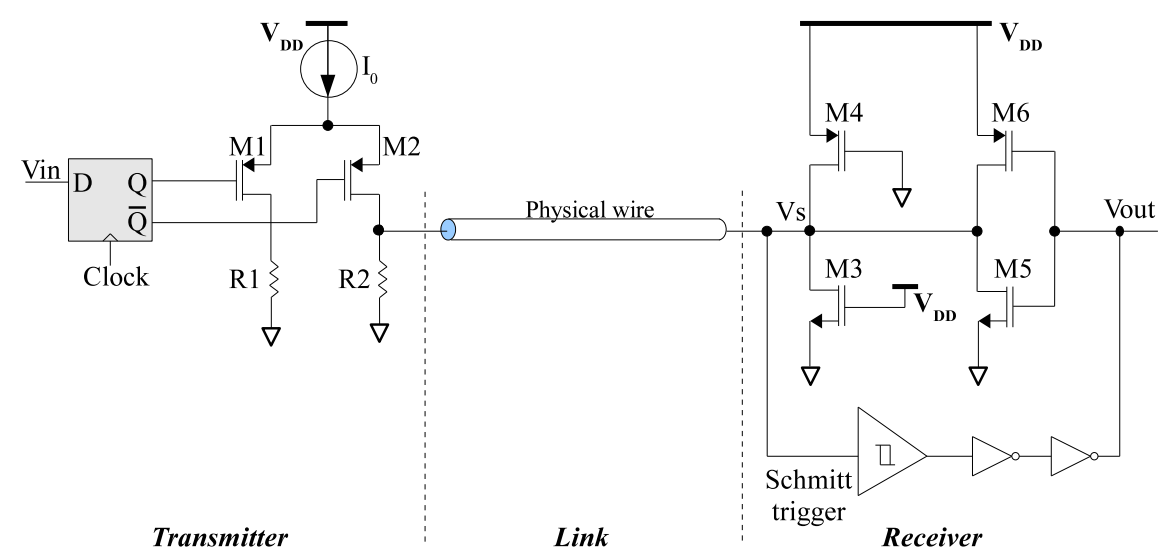

Fig. 1. Overall architecture of the proposed transceiver of the current-mode link.

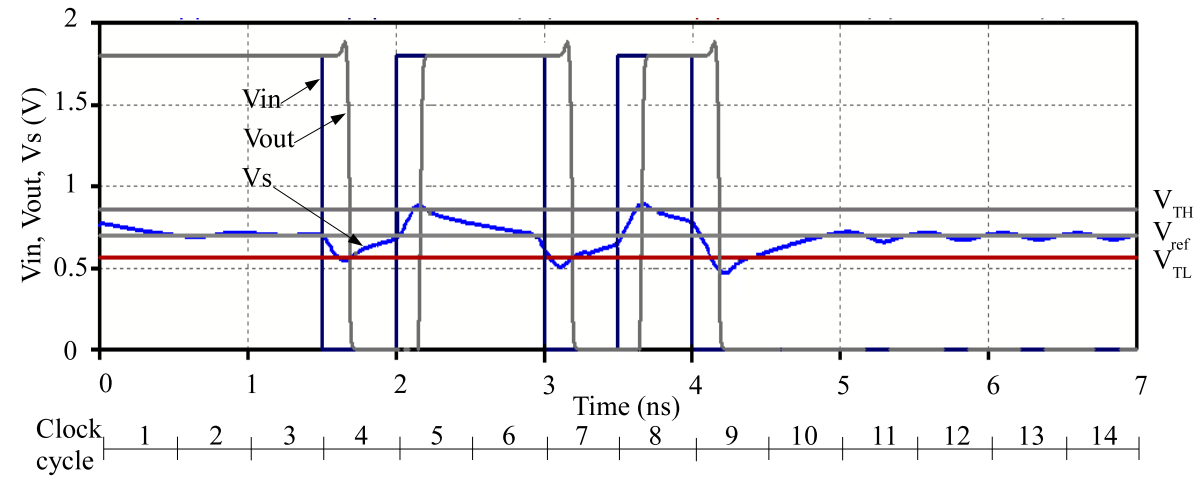

Fig. 2. Waveforms of signals of interest during normal operation of the proposed current-mode link.

whose technology parameters are estimated via the technology prediction model from [28]. We utilize Cadence Spectre [29] for our simulations. In what follows we report delay and power consumption simulation results.

The latency is defined as the difference between the time the input is applied to the transceiver and time the output signal is received. In other words, link latency includes the delay of the transmitter, the link, and the receiver from Fig. 1. The variation of the overall transceiver latency or delay versus the link length is shown in Fig.3 for a wire width of $0.36 \mu \mathrm{m}$ and the link length varied between $1 \mathrm{~mm}$ and $6 \mathrm{~mm}$. Simulations are performed under a clock period of $T_{\text {clock }}=0.5 \mathrm{~ns}$ with $1 \%$ edge rise and fall times.

The variation of the overall transceiver power consumption versus the link length is shown in Fig.4. Simulations are performed for a $V_{D D}=1.8 \mathrm{~V}$.

\section{A. Performance sensitivity to power supply and variation with technology}

The impact of the power supply changes on the performance of the proposed link is illustrated in Fig.5 and Fig.6. As expected, increasing the power supply leads to better performance but increased power consumption, while any decrease in power supply reduces the power consumption but increases the link latency.

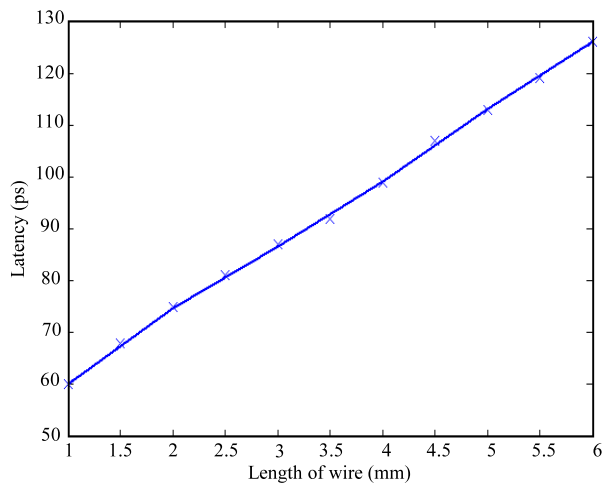

Fig. 3. Latency versus link length.

Fig.7 shows simulations results of the link latency for different CMOS technologies. In our simulations we use technology parameters reported by the predictive technology model [28]. We notice that link delay increases linearly as a result of that in more recent technologies wire resistances and capacitances increase.

\section{B. Qualitative comparison with related work}

In this section, we attempt to compare the proposed currentmode link to previous works. It is difficult to conduct a fair comparison because to quantify the advantages and disad- 


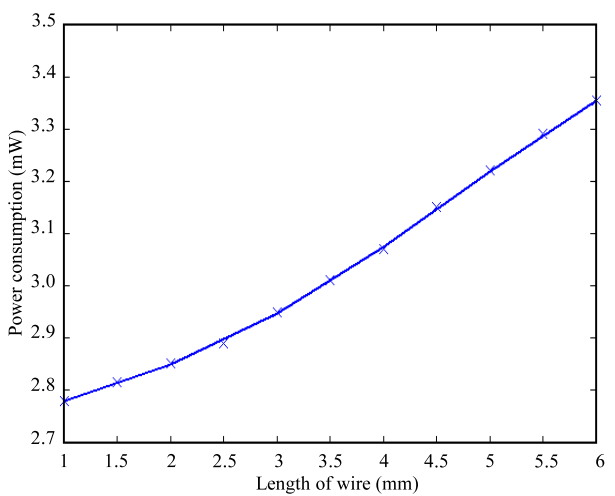

Fig. 4. Power consumption versus link length.

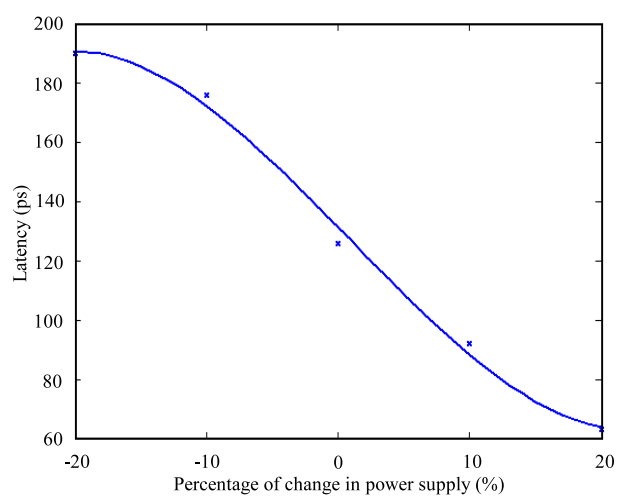

Fig. 5. Illustration of sensitivity of the measured latency results to power supply changes for $6 \mathrm{~mm}$ long link.

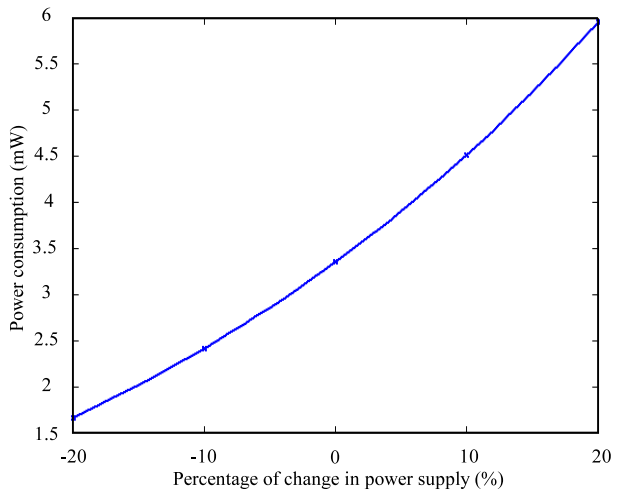

Fig. 6. Illustration of sensitivity of the measured power consumption results to power supply changes for $6 \mathrm{~mm}$ long link.

vantages of a given long-range link approach, one has to consider similar constraints. For example, if one wants to compare traditional voltage-mode links versus optical links in terms of delay and bandwidth, the comparison should be done assuming similar area and/or fabrication cost budgets. In addition, some interconnect solutions may offer features that cannot be achieved by others. For example, wireless links can transmit to multiple receivers or they can be easily reconfigured to form new routing paths. Therefore, here, we present a qualitative comparison of the proposed current-mode

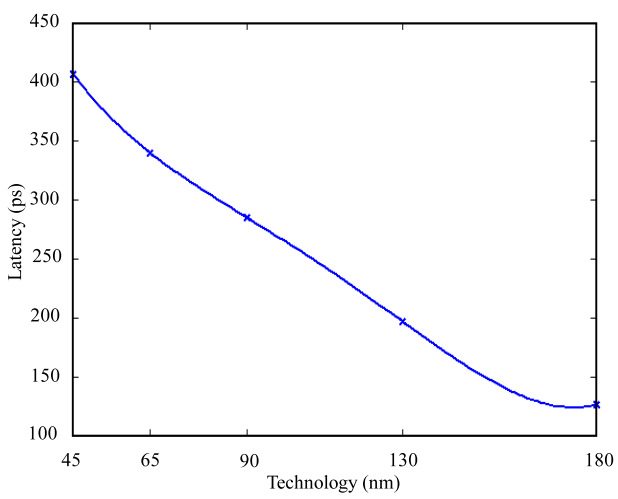

Fig. 7. Illustration of the measured latency results for different CMOS technology for $6 \mathrm{~mm}$ long link.

TABLE I

COMPARISON OF THE PROPOSED CURRENT-MODE LINK WITH OTHER CMOS CURRENT-MODE INTERCONNECTS.

\begin{tabular}{|c|c|c|c|c|}
\hline Interconnect & $\begin{array}{l}\text { Power or energy } \\
\text { consumption }\end{array}$ & Latency & $\begin{array}{l}\text { Wire } \\
\text { width }\end{array}$ & Technology \\
\hline Voltage-mode [24] & $\begin{array}{c}1.8 p J / b i t \\
\text { Wire length: } 6 \mathrm{~mm}\end{array}$ & $\approx 250 \mathrm{ps}$ & $0.5 \mu m$ & $\begin{array}{c}0.18 \mu \mathrm{m} \\
1.8 \mathrm{~V}\end{array}$ \\
\hline Current-mode [22] & $\begin{array}{c}16 \mathrm{~mW} \\
\text { Wire length: } 20 \mathrm{~mm}\end{array}$ & $\approx 300 \mathrm{ps}$ & $16 \mu m$ & $\begin{array}{c}0.18 \mu \mathrm{m} \\
1.8 \mathrm{~V}\end{array}$ \\
\hline Current-mode [23] & $\begin{array}{c}1.224 \mathrm{pJ} \\
\text { Wire length: } 6 \mathrm{~mm}\end{array}$ & $\approx 341 p s$ & $0.3 \mu \mathrm{m}$ & $\begin{array}{c}0.11 \mu \mathrm{m} \\
1.2 \mathrm{~V}\end{array}$ \\
\hline Current-mode [24] & $\begin{array}{c}0.29 \mathrm{pJ} / \mathrm{bit} \\
\text { Wire length: } 3 \mathrm{~mm}\end{array}$ & $\approx 36 \mathrm{ps}$ & $4 \mu m$ & $\begin{array}{c}0.18 \mu \mathrm{m} \\
1.8 \mathrm{~V}\end{array}$ \\
\hline Current-mode [26] & $\begin{array}{c}\approx 0.6 \mathrm{~mW} \\
\text { Wire length: } 6 \mathrm{~mm}\end{array}$ & $\approx 500 \mathrm{ps}$ & - & $\begin{array}{c}0.13 \mu \mathrm{m} \\
1.2 \mathrm{~V}\end{array}$ \\
\hline Voltage-mode [33] & $\begin{array}{c}2 p J / b i t \\
\text { Wire length: } 10 \mathrm{~mm}\end{array}$ & $440 p s$ & $0.4 \mu \mathrm{m}$ & $\begin{array}{c}0.13 \mu \mathrm{m} \\
1.2 \mathrm{~V}\end{array}$ \\
\hline Current-mode [25] & $\begin{array}{c}24 \mathrm{~mW} \\
\text { Wire length: } 7 \mathrm{~mm}\end{array}$ & - & $2 \mu m$ & $\begin{array}{c}65 \mathrm{~nm} \\
-\end{array}$ \\
\hline Proposed & $\begin{array}{c}3.355 \mathrm{~mW} \\
\text { Wire length: } 6 \mathrm{~mm}\end{array}$ & $126 p s$ & $0.36 \mu \mathrm{m}$ & $\begin{array}{c}0.18 \mu \mathrm{m} \\
1.8 \mathrm{~V}\end{array}$ \\
\hline
\end{tabular}

link with previous works.

Table I compares the proposed current-mode link with several other reported CMOS based links. The comparison is done for a link width of $0.36 \mu \mathrm{m}$ and a length of $6 \mathrm{~mm}$. We note that the proposed current-mode link outperforms or offers comparable performance to that of previously proposed links. The only previous work that reports a shorter delay is [24] however for a link length of $3 \mathrm{~mm}$ and wire width of $4 \mu \mathrm{m}$. If we use the same wire dimensions, then the proposed current-mode transceiver's performance would be $30 \mathrm{ps}$.

Table II compares the proposed current-mode link with some of the wireless and optical interconnects that were recently proposed also in the context of NoC design. We note that the proposed link is implemented completely in CMOS technology. Therefore, it requires less area and is cheaper to implement compared to the wireless and optical counterparts.

\section{ApPliCATIONS}

In this section we discuss how current-mode links are utilized to improve the performance of NoC based systems.

The concept of long-range link insertion within the context of NoCs has been proposed in [3] and it consists of the superposition of a few long-range links and a standard mesh network. Inserting application-specific long-range links enables a higher network throughput and a lower average 


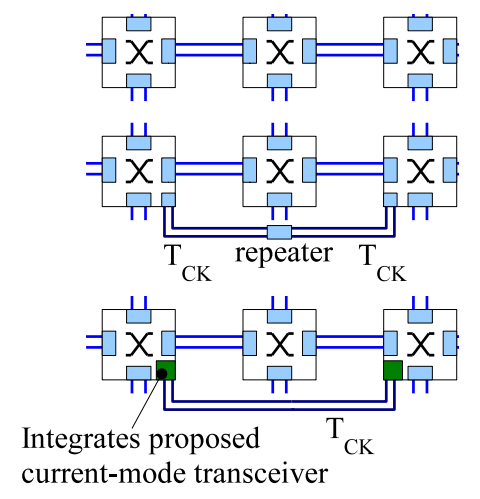

Fig. 8. Implementation of long-range links using buffered voltage-mode links or current-mode links.

TABLE II

COMPARISON OF THE PROPOSED CURRENT-MODE LINK WITH WIRELESS AND OPTICAL INTERCONNECTS.

\begin{tabular}{|c|c|c|c|c|}
\hline Interconnect & Power consumption & Latency & Area & Technology \\
\hline Wireless [7] & $\begin{array}{c}61.6 \mathrm{~mW} \\
\text { Wire length: } 1 \mathrm{~mm}\end{array}$ & - & $\begin{array}{l}\text { Transmitter: } 0.1 \mathrm{~mm}^{2} \\
\text { Receiver: } 0.54 \mathrm{~mm}^{2} \\
\text { Antenna length: } 2.98 \mathrm{~mm}\end{array}$ & $0.18 \mu \mathrm{m}$ \\
\hline Optical [15] & $\begin{array}{l}18.7 m W \\
8.8 m W \\
6.3 m W \\
5.3 m W\end{array}$ & $\begin{array}{c}162.0 \mathrm{ps} \\
113.6 \mathrm{ps} \\
90.2 \mathrm{ps} \\
74.7 \mathrm{ps} \\
\text { Wire length: } 1 \mathrm{~cm}\end{array}$ & $\begin{array}{l}- \\
- \\
- \\
-\end{array}$ & $\begin{array}{l}65 \mathrm{~nm} \\
45 \mathrm{~nm} \\
32 \mathrm{~nm} \\
22 \mathrm{~nm}\end{array}$ \\
\hline Proposed & $3.355 \mathrm{~mW}$ & $\begin{array}{c}126 \mathrm{ps} \\
\text { Wire length: } 6 \mathrm{~mm} \\
\text { Wire width: } 0.36 \mu \mathrm{m}\end{array}$ & $\begin{array}{l}0.05 \mu m^{2} \\
16 \text { bits wide link }\end{array}$ & $0.18 \mu m$ \\
\hline
\end{tabular}
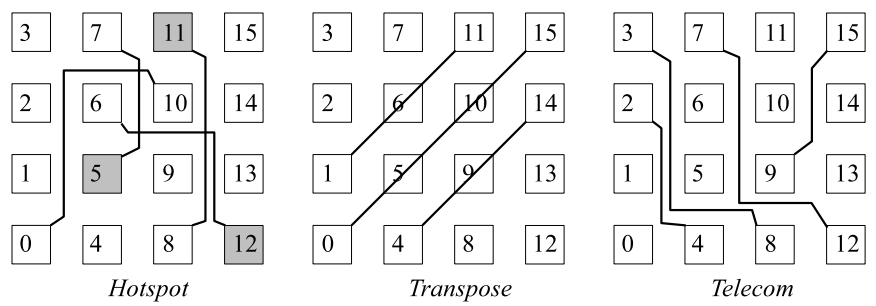

Fig. 9. Illustration of the long-range links inserted by smallnoc tool. Regular links between routers are not shown to keep figures simple. Hotspot routers are shown in grey.

packet latency compared to a simple regular mesh architecture. Therefore, long-range links can be employed to improve the performance of solutions achieved by existing NoC mapping algorithms, to achieve fault tolerance or QoS, or to trade performance improvement with power consumption reduction.

Aside from the area increase of the affected routers (which need additional input and output ports), long-range links must be buffered to be able to maintain the same global clock frequency. This is done for example in [3] by inserting repeaters, which also results in area penalty, as shown in Fig.8. However, the latency of such a long-range link depends on the number of repeaters inserted. To address this issue, we propose to implement long-range links with the current-mode links introduced in this paper. This effectively reduces the latency of each of the long-range links to just one clock period and thereby making it independent of the number of hops between the two routers connected by the link. As a result, the average packet latency of the network can be improved even further.
To investigate the above claim, we conduct simulation experiments on several $4 \times 4$ networks. In our simulations we use the smallnoc algorithm [3] to find the location of the long-range links to insert in each of the investigated networks. Information on the location of the long-range links is then utilized as part of the input given to the wormsim cycleaccurate NoC simulator, which is able to simulate regular networks as well as networks modified with long-range links. Both tools can be downloaded from [30]. Note that the smallnoc tool also calculates the content of all routing tables to guarantee deadlock free routing after the long-range link insertion. We have modified the wormsim simulator to be able to simulate also the proposed current-mode links.

In the first simulation, we study hotspot and transpose synthetic traffic workloads. For hotspot traffic, three nodes (5, 11, and 12) are selected randomly to act as hotspot nodes. Each node in the network sends packets to all other nodes. The hotspot nodes are setup such that they receive 5\% more packets on average compared to the rest of the nodes. For transpose traffic, each node communicates only with the symmetric node with respect to the diagonal of the network. In the second simulation, we evaluate the performance of the telecom benchmark, which is provided as part of the wormsim tool and which was also studied in [3]. In all our simulations we use the following settings for the wormsim simulator: packet size of one flit, input and output buffer size of two packets, and one virtual channel per input port. The remaining settings are kept to their default values. Similar to [3], we run the smallnoc tool with a constraint of $S=12$, where $S$ is the total length of all long-range links measured in terms of standard links or hops. The solutions achieved by the smallnoc tool are shown in Fig.9.

The variation of the average packet latency as a function of the packet injection rate is shown in Fig.10 for the hotspot, transpose, and telecom traffics. As expected, when long-range links are implemented as current-mode links the average packet latency improves across all values of the packet injection rate.

\section{CONClusion}

We have introduced a novel high-speed cost-effective current-mode interconnect design. Simulations demonstrate that the proposed current-mode transceiver can offer a latency of $126 \mathrm{ps}$ at a power consumption of $3.35 \mathrm{~mW}$ for an actual wire length of $6 \mathrm{~mm}$ and wire width of $0.36 \mu \mathrm{m}$ in $0.18 \mu \mathrm{m}$ CMOS technology. These results improve on the state-of-the-art current-mode links and represents a better compromise between the benefits of high speed of wireless and optical interconnects and of reduced area and cost of traditional repeater-pipelined voltage-mode interconnects. Simulations with a modified $\mathrm{NoC}$ simulator demonstrate that current-mode long-range links can improve the average flit latency.

\section{REFERENCES}

[1] W.J. Dally and B. Towles, Principles and practices of interconnection networks, Morgan Kaufmann, 2004. 


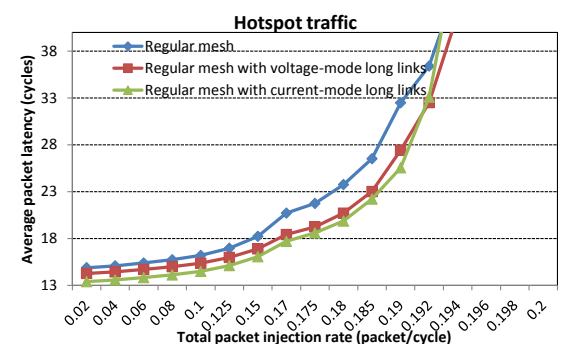

(a) Average packet latency versus traffic injection rate for hotspot traffic.

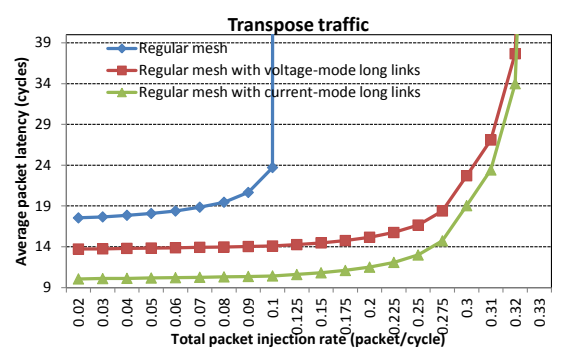

(b) Average packet latency versus traffic injection rate for transpose traffic.

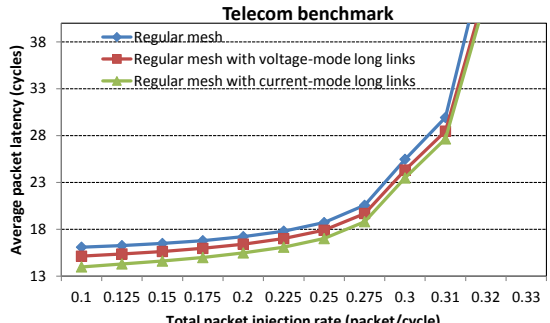

Total packet injection rate (packet/cycle)

Fig. 10. Average packet latency versus traffic injection rate

[2] G.D. Micheli and L. Benini, Networks on chips: technology and tools, Morgan Kaufmann, 2006.

[3] U.Y. Ogras and R. Marculescu, "It's a small world after all: NoC performance optimization via long-range link insertion," IEEE Trans. on Very Large Scale Integration (VLSI) Systems, vol. 14, no. 7, pp. 693-706, July 2006.

[4] M.C.F. Chang, J. Cong, A. Kaplan, C. Liu, M. Naik, J. Premkumar, G. Reinman, E. Socher, and S.W. Tam, "Power reduction of CMP communication networks via RF-interconnects," ACM/IEEE Int. Symposium on Microarchitecture, 2008.

[5] S.W. Tam, E. Socher, A. Wong, and M.C.F. Chang, "A simultaneous triband on-chip RF-interconnect for future network-on-chip," Symposium on VLSI circuits, 2009.

[6] D. Zhao, S. Upadhyaya, and M. Margala, "Design of a wireless test control network with radio-on-chip technology for nanometer systemon-a-chip," IEEE Trans. on Computer-Aided Design of Integrated Circuits and Systems (TCAD), vol. 25, no. 7, pp. 1411-1418, July 2006.

[7] D. Zhao, "Ultraperformance wireless interconnect nanonetworks for heterogeneous gigascale multi-processor SoCs," Workshop on Chip Multiprocessor Memory Systems and Interconnects (CMP-MSI'08), 2008.

[8] A. More and B. Taskin, "Simulation based study of on-chip antennas for a reconfigurable hybrid 3D wireless NoC," IEEE Int. SoC Sonference, 2010.

[9] A. Shacham, K. Bergman, and L.P. Carloni, "Photonic networks-onchip for future generations of chip multiprocessors," IEEE Trans. on Computers, vol. 57, no. 9, pp. 1246-1260, Sep. 2008.

[10] M. Petracca, K. Bergman, and L.P. Carloni, "Photonic networks-onchip: opportunities and challenges," IEEE Int. Symposium on Circuits and Systems, 2008.

[11] S. Pasricha and S. Bahirat, "OPAL: a multi-layer hybrid photonic NoC for 3D ICs," ACM/IEEE Asia and South Pacific Design Automation Conference (ASP-DAC), 2011.

[12] G. Chen, H. Chen, M. Haurylau, N. Nelson, P.M. Fauchet, E.G. Friedman, and D. Albonesi, "Predictions of CMOS compatible on chip optical interconnect," ACM/IEEE Int. Workshop on System Level Interconnect Prediction (SLIP), 2005.

[13] A.K. Kodi and A. Louri, "System simulation methodology of optical interconnects for high performance computing systems," Journal of Optical Networking, vol. 6, no. 12, pp. 1282-1300, Nov. 2007.

[14] B.G. Lee, X. Chen, A. Biberman, X. Liu, I.W. Hsieh, C.Y. Chou, J.I. Dadap, F. Xia, W.M.J. Green, L. Sekaric, Y.A. Vlasov, R.M. Osgood, and K. Bergman, "Ultrahigh-bandwidth silicon photonic nanowire waveguides for on-chip networks," IEEE Photonics Technology Letters, vol. 20, no. 6, pp. 398-400, March 2008.

[15] S. Pasricha and N. Dutt, "ORB: an on-chip optical ring bus communication architecture for multi-processor systems-on-chip," ACM/IEEE Asia and South Pacific Design Automation Conference (ASP-DAC), 2008.

[16] M. Petracca, B.G. Lee, K. Bergman, and L.P. Carloni, "Photonic NoCS: system-level design exploration," IEEE Micro Special Issue: Top Picks from Hot Interconnects, vol. 29, no. 4, pp. 74-85, 2009.

[17] A. Joshi, C. Batten, Y.J. Kwon, S. Beamer, I. Shamim, K. Asanovic, and V. Stojanovic, "Silicon-photonic clos networks for global onchip communication," ACM/IEEE Int. Symposium on Networks-onChip (NOCS), 2009.
[18] D. Ding, Y. Zhang, H. Huang, R.T. Chen, and D.Z. Pan, "O-Router: an optical routing framework for low power on-chip silicon nano-photonic integration," ACM/IEEE Design Automation Conference (DAC), 2009.

[19] G. Hendry, E. Robinson, V. Gleyzer, J. Chan, L.P. Carloni, N. Bliss, and K. Bergman, "Circuit-switched memory access in photonic interconnection networks for high-performance embedded computing," ACM/IEEE Conference on High Performance Computing (HPC), 2010.

[20] R.W. Morris and A.K. Kodi, "Power-efficient and high-performance multi-level hybrid nanophotonic interconnect for multicores," ACM/IEEE Int. Symposium on Networks-on-Chip (NOCS), 2010.

[21] G. Hendry, J. Chan, L.P. Carloniz, and K. Bergman, "VANDAL: a tool for the design specification of nanophotonic networks," ACM/IEEE Design Automation and Test in Europe (DATE), 2011.

[22] R.T. Chang, N. Talwalkar, C.P. Yue, and S.S. Wong, "Near speed-oflight signaling over on-chip electrical interconnects," IEEE Journal of Solid-State Circuits, vol. 38, no. 5, pp. 834-837, May 2003.

[23] N. Tzartzanis and W.W. Walker, "Differential current-mode sensing for efficient on-chip global signaling," IEEE Journal of Solid-State Circuits, vol. 40, no. 11, pp. 2141-2147, Nov. 2005.

[24] A.P. Jose, G. Patounakis, and K.L. Shepard, "Pulsed current-mode signaling for nearly speed-of-light intrachip communication," IEEE Journal of Solid-State Circuits, vol. 41, no. 4, pp. 772-780, Apr. 2006.

[25] R.R. Dobkin, M. Moyal, A. Kolodny, and R. Ginosar, "Asynchronous current mode serial communication," IEEE Trans. on Very Large Scale Integration (VLSI) Systems, vol. 18, no. 7, pp. 1107-1117, Jul. 2010.

[26] E. Nigussie, T. Lehtonen, S. Tuuna, J. Plosila, and J. Isoaho, "Highperformance long NoC link using delay-insensitive current-mode signaling," VLSI Design, 2007.

[27] F. Yuan, "CMOS current-mode circuits for data communications," Springer Science, 2010.

[28] Predictive Technology Model (PTM), Arizona State University, 2011, http://ptm.asu.edu

[29] Spectre Circuit Simulator, http://www.cadence.com/products/cic/ spectre_circuit/pages/default.aspx

[30] System Level Design Group, Software, 2011, http://www.ece.cmu.edu/ $\sim$ sld/wiki/doku.php?id=shared:software

[31] Y. Tsividis and C. McAndrew, Operation and Modeling of the MOS Transistor, Oxford University Press, 2010.

[32] Jacob Baker, CMOS Circuit Design, Layout, and Simulation, WileyIEEE Press, 2004.

[33] D. Schinkel, E. Mensink, E.A.M. Klumperink, E. van Tuijl, and B. Nauta, "A 3-Gb/s/ch Transceiver for 10-mm Uninterrupted RC-Limited Global On-Chip Interconnects," IEEE Journal of Solid-State Circuits, vol. 41, no. 1, Jan. 2006. 